\title{
Photon production and elliptic flow from a momentum-anisotropic quark-gluon plasma
}

\author{
Babak S. Kasmaei (1) and Michael Strickland (D) \\ Department of Physics, Kent State University, Kent, Ohio 44242, USA
}

(Received 25 November 2019; accepted 2 July 2020; published 22 July 2020)

\begin{abstract}
The emission of real photons from a momentum-anisotropic quark-gluon plasma (QGP) is affected by both the collective flow of the radiating medium and the modification of the local rest frame emission rate due to the anisotropic momentum distribution of partonic degrees of freedom. In this paper, we first calculate the photon production rate from an ellipsoidally momentum-anisotropic QGP including hard contributions from Compton scattering and quark pair annihilation and soft contribution calculated using the hard thermal loop approximation. We introduce a parametrization of the nonequilibrium rate in order to facilitate its further application in yield and flow calculations. We convolve the anisotropic photon rate with the space-time evolution of QGP provided by $3+1 \mathrm{~d}$ anisotropic hydrodynamics to obtain the yield and the elliptic flow coefficient $v_{2}$ of photons from QGP generated at $\mathrm{Pb}-\mathrm{Pb}$ collisions at the LHC at 2.76 TeV and $\mathrm{Au}-\mathrm{Au}$ collisions at the RHIC at $200 \mathrm{GeV}$. We investigate the effects of various parameters on the results. In particular, we analyze the sensitivity of the results to initial momentum anisotropy.
\end{abstract}

DOI: 10.1103/PhysRevD.102.014037

\section{INTRODUCTION}

One of the challenges in developing a more complete picture of the dynamics of the many-body system generated at heavy-ion collision experiments is the lack of reliable information about the initial conditions of the system due to the strong interactions among the degrees of freedom, relevance of many effects at extreme conditions, and the loss of information in a pseudothermalized system. The idea that internally generated electromagnetic probes of the strongly interacting matter can provide less distorted, or at least extra, information about the phases and evolution dynamics of the system has been suggested since the introduction of the notion of quark-gluon plasma [1,2] and has been developed through the decades by many researchers. In particular, real photons produced in heavyion collisions can be emitted from different sources and stages and are affected by various characteristics of the QCD matter [3-51]. In recent years, phenomenological studies of photon emission using hydrodynamic modeling of heavy-ion collisions have been developing [52-62] toward connecting theoretical ideas to experimental data. However, a simultaneous quantitative description of photon yield and flow coefficients has been challenging [6]. Current levels of uncertainty in experimental data of real photons at

Published by the American Physical Society under the terms of the Creative Commons Attribution 4.0 International license. Further distribution of this work must maintain attribution to the author(s) and the published article's title, journal citation, and DOI. Funded by SCOAP . the RHIC [63-65] and LHC [66] also limits the extent of reliable interpretations of theoretical predictions.

One important feature of nonequilibrium quark-gluon plasma (QGP), as suggested by different microscopic models and successful phenomenological studies, is the anisotropy of the local rest frame (LRF) momentum distributions for partonic degrees of freedom [67]. Development of relativistic anisotropic hydrodynamics [68-73] has allowed for consistent incorporation of momentum anisotropy in the collective dynamics of QGP. The higher accuracy of the anisotropic hydrodynamics (aHydro) model in the description of nonequilibrium dynamics compared to other hydrodynamical models and with reference to various exact solutions has been discussed in several papers [72,74-79]. Modification of the photon production rate due to momentum anisotropy has also been studied [51] using the parametrization introduced in [80] where momentum anisotropy is described by the spheroidal deformation of conventional isotropic distributions. Using the photon rate and an early version of anisotropic hydrodynamics using a spheroidal parametrization, the yield and $v_{2}$ of photons from QGP has been studied previously [55]. In this paper, for first time we include in the LRF photon rate calculation the momentum anisotropy in the transverse direction in the form of ellipsoidal deformation of isotropic distributions. This is done using an efficient method introduced in our previous paper [81] to calculate the quark self-energies in anisotropic medium. In this paper, we further introduce a suitable ansatz to encode the numerically calculated nonequilibrium photon rate values in a compact form which facilitates its use for hydrodynamic computation of photon yield and flow. 
We convolve the LRF photon rate with the state-of-the-art $3+1 \mathrm{~d}$ relativistic anisotropic hydrodynamic model with a quaisparticle equation of state (EOS) [72] and obtain results for the photon yield and $v_{2}$. We report the results for different centrality classes, initial momentum anisotropies, $\mathrm{Pb}-\mathrm{Pb}$ collisions at the LHC, and Au-Au collision at the RHIC. We also investigate the uncertainties due to our approximations in using aHydro output for photon rate calculations.

\section{PHOTON PRODUCTION RATE}

In the local rest frame of a QGP fluid element, we consider the production rate of real photons from the hard processes of Compton scattering $q g \rightarrow q \gamma$ and pair annihilation $q \bar{q} \rightarrow g \gamma$ calculated at tree level and from soft processes calculated within hard thermal loop perturbation theory [82] at leading order. The photon production rate is then the combination of hard and soft contributions separated at a momentum scale $p^{*}$ which serves as an IR/UV cutoff for hard/soft processes. With nonequilibrium momentum distributions $f_{q / g}(\mathbf{k})$ for partonic degrees of freedom, the production rate of photons with momentum $q$ from Compton scattering is

$$
\begin{aligned}
q \frac{d R_{\mathrm{Com}}^{\gamma}}{d^{3} q}= & -128 \pi^{3} \alpha_{s} \alpha_{\mathrm{em}} \sum_{j \in\{u, d\}} e_{j}^{2} \int_{\mathbf{k}_{\mathbf{1}}} \frac{f_{q}\left(\mathbf{k}_{\mathbf{1}}\right)}{k_{1}} \\
& \times \int_{\mathbf{k}_{2}} \frac{f_{g}\left(\mathbf{k}_{\mathbf{2}}\right)}{k_{2}} \int_{\mathbf{k}_{3}} \frac{1-f_{q}\left(\mathbf{k}_{\mathbf{3}}\right)}{k_{3}} \\
& \times \delta^{4}\left(K_{1}-K_{2}-K_{3}-Q\right)\left[\frac{s}{t}+\frac{t}{s}\right],
\end{aligned}
$$

and the rate from the annihilation process is calculated as

$$
\begin{aligned}
q \frac{d R_{\mathrm{Ann}}^{\gamma}}{d^{3} q}= & 64 \pi^{3} \alpha_{s} \alpha_{\mathrm{em}} \sum_{j \in\{u, d\}} e_{j}^{2} \int_{\mathbf{k}_{1}} \frac{f_{q}\left(\mathbf{k}_{\mathbf{1}}\right)}{k_{1}} \\
& \times \int_{\mathbf{k}_{2}} \frac{f_{q}\left(\mathbf{k}_{\mathbf{2}}\right)}{k_{2}} \int_{\mathbf{k}_{\mathbf{3}}} \frac{1+f_{g}\left(\mathbf{k}_{\mathbf{3}}\right)}{k_{3}} \\
& \times \delta^{4}\left(K_{1}-K_{2}-K_{3}-Q\right)\left[\frac{u}{t}+\frac{t}{u}\right],
\end{aligned}
$$

where $s, t$, and $u$ are usual Mandelstam variables. In this paper, we use $\alpha_{s}=0.3$ and $\alpha_{\mathrm{em}}=1 / 137$. The IR cutoff $p^{*}$ is imposed on the momentum transfer $P=K_{1}-Q$ of hard Compton and annihilation processes.

The soft contribution to the photon rate is given by [37]

$$
q \frac{d R_{\text {Soft }}^{\gamma}}{d^{3} q}=\frac{i}{2(2 \pi)^{3}} \operatorname{Tr} \Pi_{12}(Q),
$$

where the hard loop result for the trace of the (12) element of the photon polarization tensor is $i \operatorname{Tr} \Pi_{12}(Q)=-\sum_{j \in\{u, d\}} 8 e^{2} e_{j}^{2} N_{c} \frac{f_{q}(\mathbf{q})}{q} \int_{\mathbf{p}}^{p^{*}} Q_{\nu} \tilde{W}^{\nu}(\mathbf{p})$,

where $p^{*}$ acts as the UV cutoff for the integration and we have defined [51]

$$
\begin{array}{r}
\tilde{W}^{\nu}(\mathbf{p})=\left[W^{\nu \alpha}{ }_{\alpha}(P)-W_{\alpha}^{\nu \alpha}(P)+W_{\alpha}^{\alpha \nu}(P)\right]_{p_{0}=p(\hat{\mathbf{p}} . \hat{\mathbf{q}})}, \\
W_{\alpha \beta \gamma}=\frac{P_{\alpha}-\Sigma_{\alpha}(P)}{(P-\Sigma(P))^{2}} \operatorname{Im}\left[\Sigma_{\beta}(P)\right] \frac{P_{\gamma}-\Sigma_{\gamma}^{*}(P)}{\left(P-\Sigma^{*}(P)\right)^{2}},
\end{array}
$$

and the quark self-energy is given by

$$
\Sigma(P)=\frac{C_{F}}{4} g_{s}^{2} \int_{\mathbf{k}} \frac{\hat{f}(\mathbf{k})}{|\mathbf{k}|} \frac{K \cdot \gamma}{K . P},
$$

in which the combined distribution $\hat{f}(\mathbf{k})=4 f_{\mathrm{g}}(\mathbf{k})+$ $2\left(f_{\mathrm{q}}(\mathbf{k})+f_{\bar{q}}(\mathbf{k})\right)$ is used [83].

The photon rate for anisotropic momentum distributions with spheroidal parametrization has been calculated previously [51]. In this paper, we extend the results to include an ellipsoidally anisotropic distribution in the LRF

$$
f(\mathbf{k})=f_{\text {iso }}\left(\frac{k}{\Lambda} \sqrt{1+\xi_{1}\left(\hat{\mathbf{k}} . \hat{\mathbf{n}}_{\mathbf{1}}\right)^{2}+\xi_{2}\left(\hat{\mathbf{k}} . \hat{\mathbf{n}}_{\mathbf{2}}\right)^{2}}\right),
$$

with which the photon rate acquires dependence on two more variables $\xi_{2}$ and $\phi_{q} . \Lambda$ is the temperaturelike scale. In some equations or plots, we represent momenta as scaled by $\Lambda$, i.e., $\hat{q}=q / \Lambda$. For $f_{\text {iso }}$, Fermi-Dirac/Bose-Einstein distribution is used for quarks/gluons.

The generalization of hard contributions to the ellipsoidal case is straightforward. On the contrary, calculation of the quark self-energy (7) for an ellipsoidal anisotropy was shown [84] to be more tedious than the spheroidal case [85]. In a previous paper [81], we introduced an efficient method to calculate the integral (7) for general forms of anisotropic momentum distributions which makes it possible to obtain the results for the photon rates in this paper.

The total photon rate calculated with the separation of hard and soft momenta (Braaten-Yuan method [86]) depends on the cutoff $p^{*}$. We select the point for which the total rate as a function of $p^{*}$ has its minimum. The motivation for this choice is the first order insensitivity to the separation scale $p^{*}$ of the total photon rate at the minimum $\left(d R^{\gamma} / d p^{*}=0\right)$, and also the matching of the numerical result with the known analytic approximation for the isotropic and small coupling limit $[9,51,55]$. This idea is shown in the left panel of Fig. 1 for an example set of parameters where we fit a polynomial to the numerically calculated points and then estimate the minimal point. In cases where the polynomial fitting was not accurate enough, we selected the minimum value among the list of numerical results. By selecting points away from the 


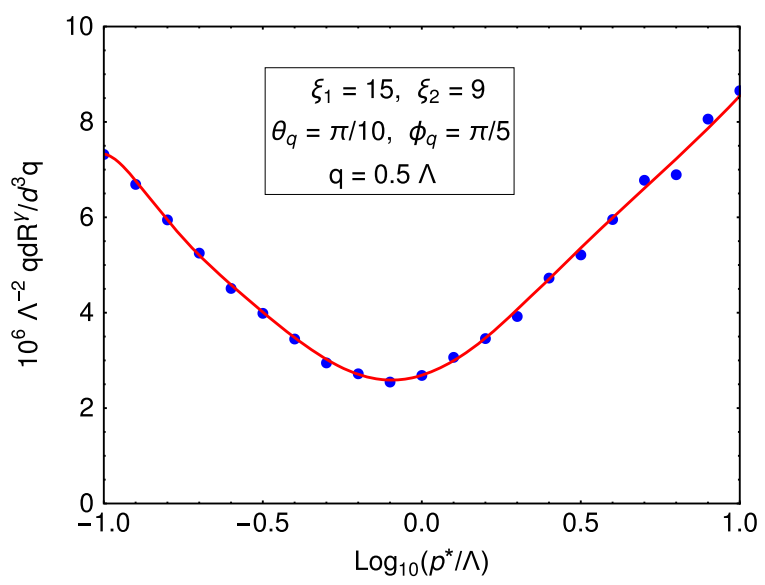

(a)

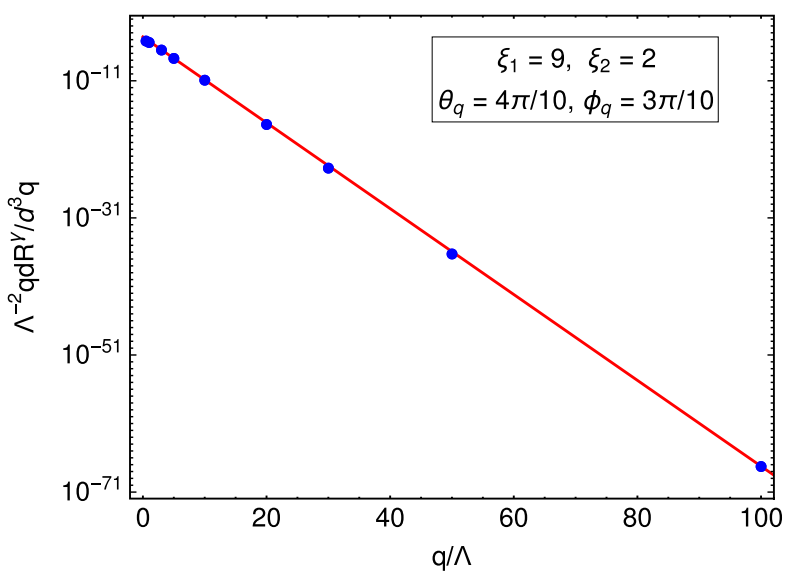

(b)

FIG. 1. (a) Example case for the total photon rate as a function of the hard/soft separation momentum $p^{*}$. Points represent numerically calculated results and the solid curve is a fitted polynomial to the points. (b) An example case for fitting the exponential ansatz (9) to the numerically calculated values of the photon rate.

estimated minimal point, we also checked that the uncertainty of the photon rate value due to this variation is small.

For the purpose of calculating photon yield/flow, the values of the production rate with different parameters and for all space-time points of QGP evolution need to be obtained. The procedure of evaluating hard and soft contribution integrals then finding the minimum point is too time consuming to be performed repeatedly in order to calculate the photon yield/flow. Therefore, we first obtain and tabulate the photon production rates for a large set of different values for parameters $\left\{\xi_{1}, \xi_{2}, \theta_{q}, \phi_{q}, q\right\}$. For each pair of $\left\{\xi_{1}, \xi_{2}\right\}$, we fit the corresponding tabulated results to the ansatz

$\hat{q} \frac{d R^{\gamma}}{d^{3} \hat{q}}=\exp \left[-\alpha\left(\theta_{q}, \phi_{q} ; \xi_{1}, \xi_{2}\right)-\beta\left(\theta_{q}, \phi_{q} ; \xi_{1}, \xi_{2}\right) \hat{q}\right]$

and

$$
\begin{aligned}
\alpha\left(\theta_{q}, \phi_{q} ; \xi_{1}, \xi_{2}\right)= & \sum_{m=0}^{6} \sum_{n=0}^{6} a_{m n}\left(\xi_{1}, \xi_{2}\right)\left(\operatorname{sgn}\left(\xi_{1}\right) \cos \theta_{q}\right)^{2 m} \\
& \times\left(\operatorname{sgn}\left(\xi_{2}\right) \cos \phi_{q}\right)^{2 n}, \\
\beta\left(\theta_{q}, \phi_{q} ; \xi_{1}, \xi_{2}\right)= & \sum_{m=0}^{6} \sum_{n=0}^{6} b_{m n}\left(\xi_{1}, \xi_{2}\right)\left(\operatorname{sgn}\left(\xi_{1}\right) \cos \theta_{q}\right)^{2 m} \\
& \times\left(\operatorname{sgn}\left(\xi_{2}\right) \cos \phi_{q}\right)^{2 n},
\end{aligned}
$$

where the sign function $\operatorname{sgn}(x)$ is used to prevent extra $\theta_{q} / \phi_{q}$ dependence of the fitted function for $\xi_{1} / \xi_{2}=0$ due to numerical artifacts. We make a lookup table of coefficients $a_{m n}$ and $b_{m n}$ for each point on the grid of $\left\{\xi_{1}(i), \xi_{2}(j)\right\}$. The photon production rate for the $\left\{\xi_{1}, \xi_{2}\right\}$ values at the grid points will be calculated using (9)-(11). For other values of $\left\{\xi_{1}, \xi_{2}\right\}$, we use a linear interpolation of the $\log \left(\hat{q} \frac{d R^{\gamma}}{d^{3} \hat{q}}\right)$ values at the nearest points of the grid to $\left\{\xi_{1}, \xi_{2}\right\}$.

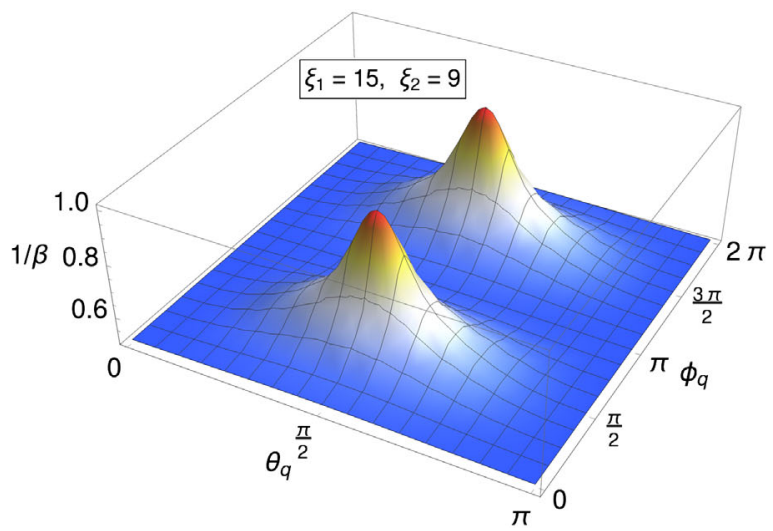

FIG. 2. Angular dependence of $1 / \beta$ of ansatz (9) for two example cases. 
In the right panel of Fig. 1, the fitting of numerically calculated photon rates to the function $\exp (-\alpha-\beta \hat{q})$ is shown for the example case of $\left\{\xi_{1}=9, \xi_{2}=2, \theta_{q}=4 \pi / 10\right.$, $\left.\phi_{q}=3 \pi / 10\right\}$ resulting in $\exp (-10.5825-1.44214 \hat{q})$ with $R_{\text {fit }}^{2}=0.999951$. The uncertainty band due to variation of selected separation scale $p^{*}$ was not observable in the plot.

The parameter $\beta\left(\theta_{q}, \phi_{q} ; \xi_{1}, \xi_{2}\right)$ can be seen as an anisotropic rescaling factor for the inverse temperature of the radiating QGP element. One can consider $\Lambda\left(\xi_{1}, \xi_{2}\right) / \beta\left(\theta_{q}, \phi_{q} ; \xi_{1}, \xi_{2}\right)$ as the anisotropic radiation temperature of the QGP element in the local rest frame. For isotropic QGP $\beta=1$ and for small values of anisotropy parameters, it is proportional to the original anisotropic deformation kernel $\sqrt{1+\xi_{1} \cos ^{2} \theta+\xi_{2} \sin ^{2} \theta \cos ^{2} \phi}$ of QGP distributions. However, in general, especially for larger anisotropy, the relation of $\alpha$ and $\beta$ to the anisotropic form of QGP distributions is nontrivial and complicated. In Fig. 2, the factor $1 / \beta$ as a function of $\left(\theta_{q}, \phi_{q}\right)$ is shown for $\left\{\xi_{1}=12, \xi_{2}=-0.2\right\}$.

\section{HYDRODYNAMIC EVOLUTION OF QGP}

In order to calculate the yield and elliptic flow coefficient of real photons emitted from the QGP, we convolve the LRF anisotropic photon production rate with the space-time evolution of the strongly interacting fluid provided by $3+1 \mathrm{~d}$ aHydro with a quasiparticle equation of state and smooth Glauber initial conditions [70,71]. The method is basically the same as the convolution of dilepton production with aHydro evolution described in [87]. We use the aHydro parameter values tuned to reproduce soft hadrons spectra for the LHC [70] and RHIC [73]. The tuned values of the initial central temperature $T_{0}$ and shear viscosity to entropy ratio $\eta / s$ are $\{600 \mathrm{MeV}, 2 / 4 \pi\}$ for the LHC and $\{455 \mathrm{MeV}, 2.25 / 4 \pi\}$ for the RHIC. In all of the results presented in this paper, we only consider real photons with rapidity $y=0$ in the lab frame. We only consider the photons emitted from the QGP phase, and we set the rate to zero for fluid elements with effective temperature $T_{\text {eff }}$ below the critical temperature $T_{c}=155 \mathrm{MeV}$.

The evolving momentum distributions in the latest version of $3+1 \mathrm{~d}$ aHydro include a temperature-dependent mass $\hat{m}$ which is calculated using isotropic lattice QCD results and matching energy densities of isotropic and anisotropic systems. To translate the aHydro results to ellipsoidal distributions used for quark self-energy and photon rate calculations we neglect $\hat{m}$. This approximation, which we later check, allows one to convert the aHydro parameters $\left\{\alpha_{x, y, z}, \lambda\right\}$ to the parameters $\left\{\xi_{1,2}, \Lambda\right\}$ used in the photon rate by transformation

$$
\begin{gathered}
\Lambda=\lambda \alpha_{y}, \\
\xi_{1}=\left(\frac{\alpha_{y}}{\alpha_{z}}\right)^{2}-1, \\
\xi_{2}=\left(\frac{\alpha_{y}}{\alpha_{x}}\right)^{2}-1,
\end{gathered}
$$

where $\lambda$ and $\Lambda$ are temperaturelike scales of anisotropic distributions in the two parametrizations.

To illustrate the evolution of momentum anisotropy, in Fig. 3 we plot the spatial average $\left\langle\xi_{1}\right\rangle$ at the zero space-time rapidity hypersurface as a function of the proper time $\tau$. The bands in the plots show the spatial standard deviation of the $\xi_{1}$ values. We compare the evolution for cases with and without initial momentum anisotropy where both cases are tuned to give the best and similarly accurate fits to the LHC soft hadronic spectra [87]. The curves of $\left\langle\xi_{1}\right\rangle$ and its deviation in both cases converge at late times, but in the case with a relatively small initial anisotropy $\xi_{1}\left(\tau_{0}\right)=3$, the values of $\xi_{1}$ grow to much larger values at early times. Even in the initially isotropic case, nonequilibrium dissipative effects lead to growth of the momentum anisotropy before its relaxation back toward isotropy.
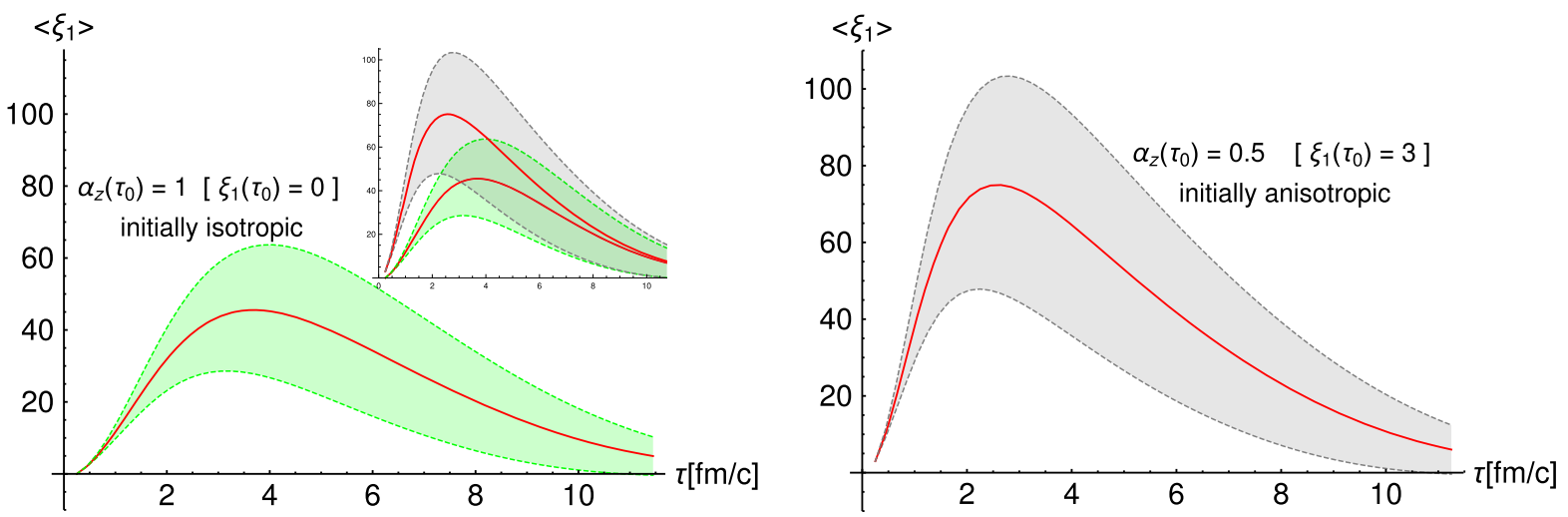

FIG. 3. Proper time evolution of the spatial average of $\xi_{1}$ values at the zero space-time rapidity hypersurface, for initially isotropic (left panel) and initially anisotropic (right panel) cases both for $30 \%-40 \% \mathrm{~Pb}-\mathrm{Pb}$ collisions. Bands represent spatial standard deviations. The inset in the left panel compares the two cases in one plot. 

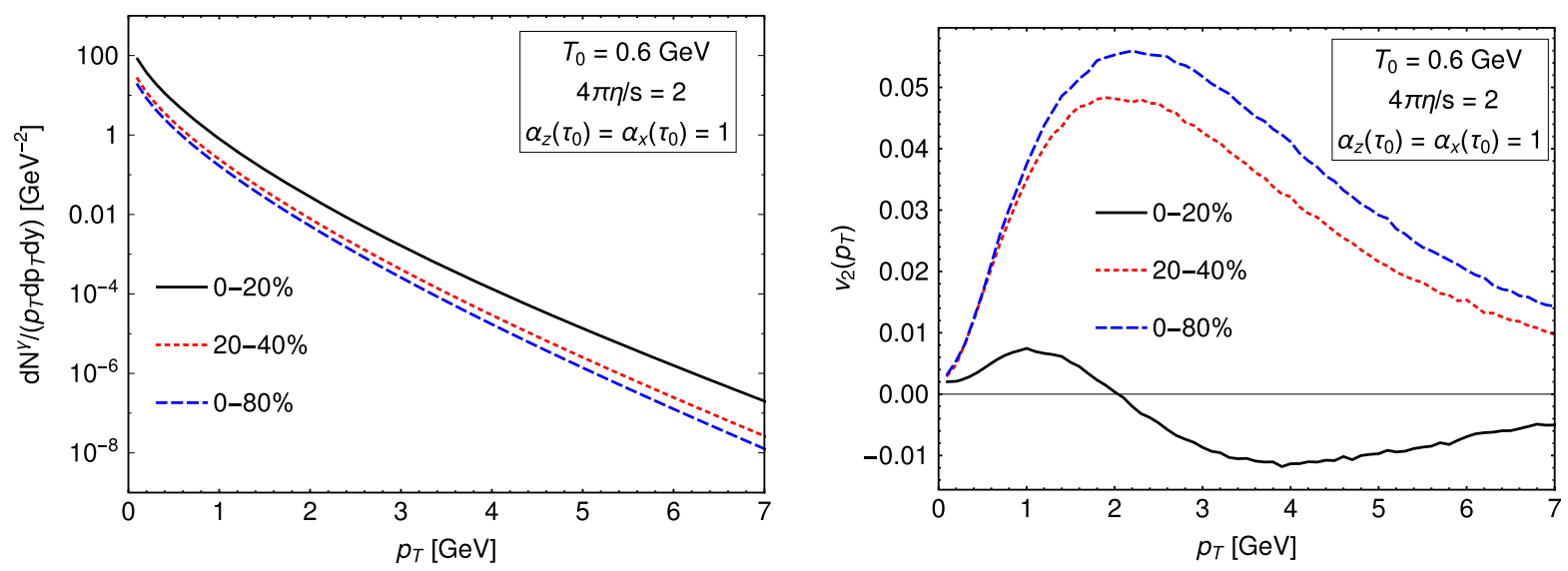

FIG. 4. Yield (left panel) and elliptic flow coefficient $v_{2}$ (right panel) of zero rapidity photons emitted from QGP generated at $\mathrm{Pb}-\mathrm{Pb}$ collisions at LHC energy. Results are shown for three centrality classes. Initial momentum isotropy is assumed.

\section{RESULTS AND DISCUSSION}

In this section, we present the results for the yield and elliptic flow coefficient $\left(v_{2}\right)$ of real photons emitted from a momentum-anisotropic QGP with space-time evolution described by aHydro. In particular, we investigate the effects of initial momentum anisotropy in both longitudinal and transverse directions.

\section{A. Different centrality classes}

The results for $\mathrm{Pb}-\mathrm{Pb}$ collisions at $\sqrt{s}=2.76 \mathrm{TeV}$, considering momentum-isotropic initial condition, are shown in Fig. 4 for the $0 \%-20 \%, 20 \%-40 \%$, and $0 \%-80 \%$ centrality classes. The QGP photon yield in central collisions is several times higher than in peripheral ones, but their $v_{2}$ shows a change in sign and is negative for $p_{T}>$ $2 \mathrm{GeV}$. The same behavior at central collisions was found for dilepton $v_{2}$ in our previous paper [87]. The origin of negative photon $v_{2}$ from anisotropic QGP can be understood as the result of the interplay between two sources of momentum anisotropy of the generated particles: (1) the collective flow of the strongly interacting medium and (2) the anisotropic rate of photon production in LRF of each fluid element. For instance, with a positive value of $\xi_{2}$, the LRF photon rate at the $\theta_{q}=0$ plane acquires a negative coefficient of $\cos \left(2 \phi_{q}\right)$ in its Fourier decomposition. In central collisions, this effect can dominate over the smaller positive elliptic flow from the fluid expansion. Most of the effects from LRF anisotropic distributions can play a role at earlier stages when distributions are further from equilibrium, and this affects mostly the particles generated with higher $p_{T}$.

We also compare with the results from the ideal limit of the hydrodynamic model. To take this limit, one needs to set the viscosity-to-entropy ratio to a very small value [69]. We set $4 \pi \eta / s=0.05$ and check that the values of anisotropic parameters $\xi_{i}$ remain close to zero through the spacetime evolution of the QGP. The results for the yield and $v_{2}$ of photons emitted from a QGP simulation at this isotropic limit and for $\mathrm{Pb}-\mathrm{Pb}$ collisions at $\sqrt{s}=2.76 \mathrm{TeV}$ are shown in Fig. 5. Comparing to Fig. 4, the ideal limit results in a smaller yield and larger $v_{2}$ which is due to the lack of
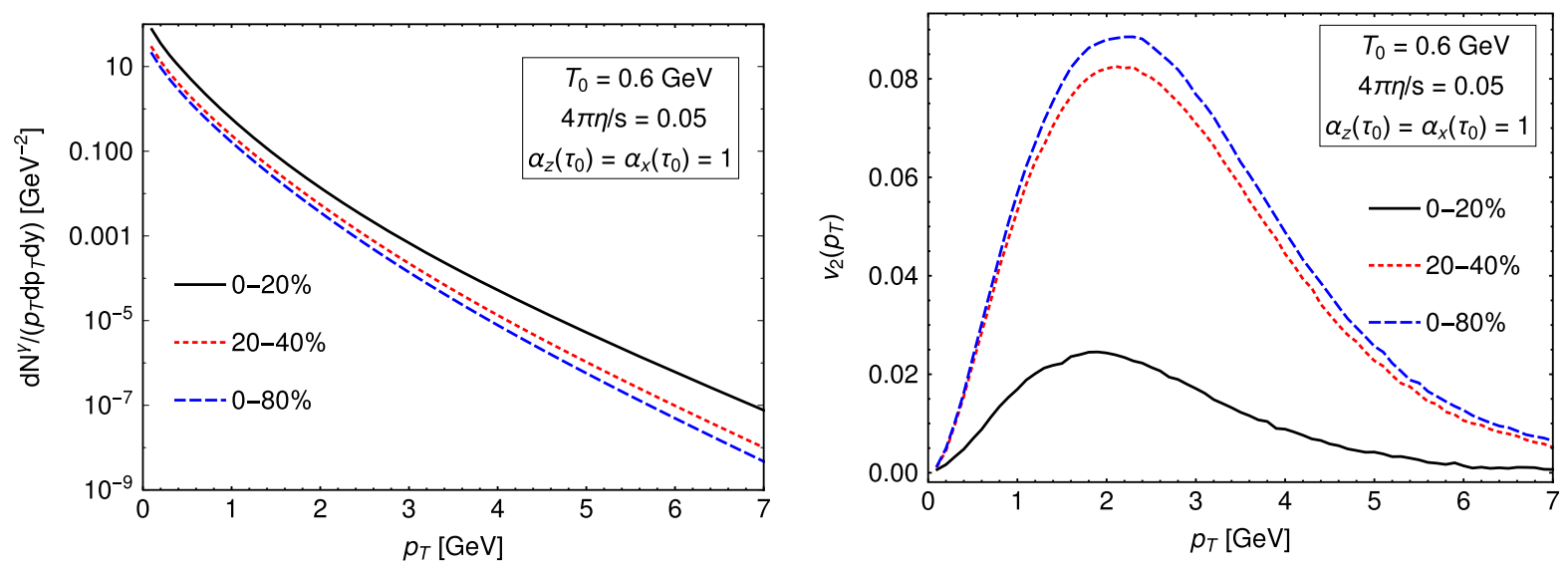

FIG. 5. Same as Fig. 4 but for the ideal (isotropic) limit of aHydro using a very small viscosity-to-entropy ratio. 

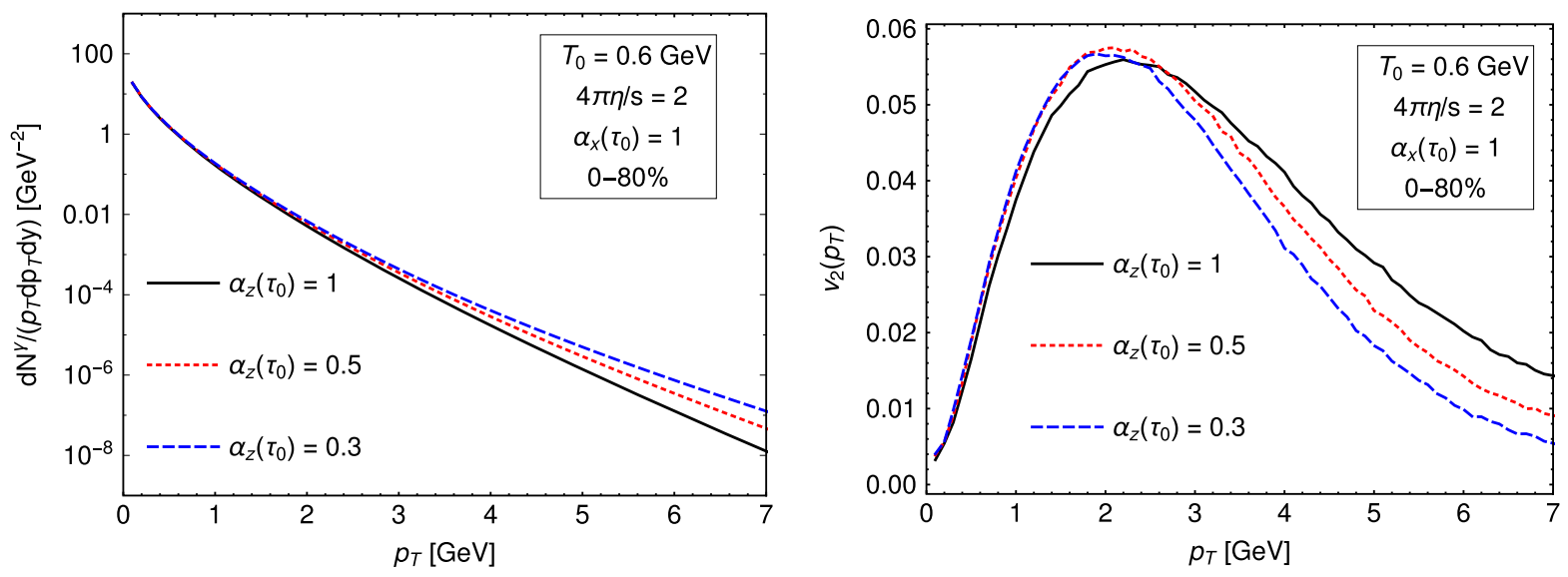

FIG. 6. Yield (left) and $v_{2}$ (right) of QGP photons at $0 \%-80 \% \mathrm{~Pb}-\mathrm{Pb}$ collisions for different initial longitudinal momentum anisotropies. Initial transverse momentum is assumed to be isotropic.

dissipative effects. The $v_{2}$ is always positive for the ideal case since the momentum distribution functions remain isotropic and do not reduce the contributions from the fluid flow.

\section{B. Varying $\alpha_{z}\left(\tau_{0}\right)$}

To investigate the effects of initial momentum anisotropy of QGP, we vary the value of $\alpha_{z}\left(\tau_{0}\right)$ from 1 (isotropic case) keeping the initial transverse momentum distribution isotropic $\left[\alpha_{x}\left(\tau_{0}\right)=1\right]$. For the $0 \%-80 \%$ centrality class, the photon yield and $v_{2}$ results with $\alpha_{z}\left(\tau_{0}\right) \in\{1,0.5,0.3\}$ are shown in Fig. 6. It is observed that the results for $p_{T} \gtrsim$ $2 \mathrm{GeV}$ are sensitive to initial momentum anisotropy. In general, the later time dynamics of the system is almost insensitive to the details of initial momentum distributions (this can also be observed from the convergence of two bands in Fig. 3). Therefore, variation of initial momentum anisotropy shows no effect on the behavior of the plots for $p_{T} \lesssim 2 \mathrm{GeV}$ which is mostly sensitive to the late-time dynamics. The yield is increased and $v_{2}$ reduces for more anisotropic momentum distributions of initial conditions

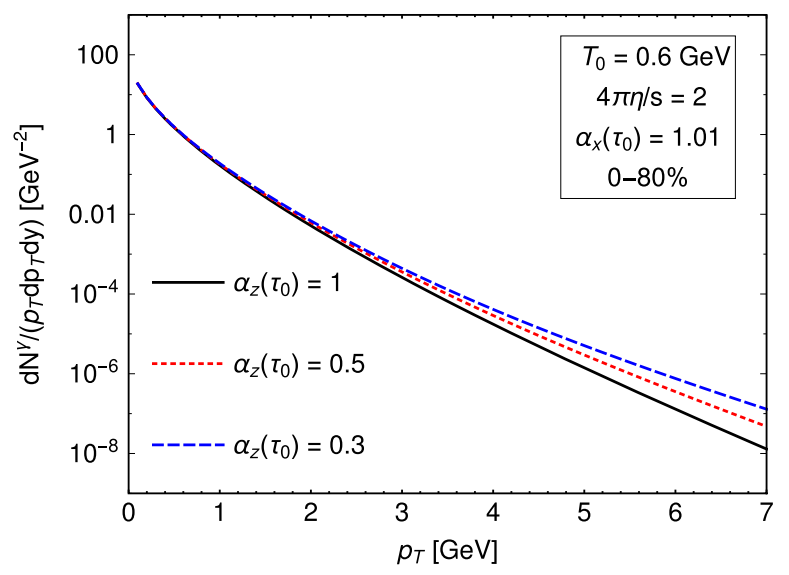

[lower $\alpha_{z}\left(\tau_{0}\right)$ ]. It should be noted that with a variation of initial momentum anisotropy, one in general needs to retune the parameters of the model such as initial temperature to find the optimal agreement with the hadronic data. In this paper, the goal is not to provide phenomenological parameter tuning, and we used the same $\left\{T_{0}, \eta / s\right\}$ for cases with different initial momentum anisotropy. In a previous paper [87], we showed that retuning $T_{0}$ for an initially anisotropic QGP does not change the interpretation of the results on electromagnetic emission.

\section{Varying $\alpha_{x}\left(\tau_{0}\right)$}

One can expect that LRF transverse momentum anisotropy can have observable effects on flow coefficients. Here we repeat the same yield and $v_{2}$ calculations as in Sec. IV B, but setting $\alpha_{x}\left(\tau_{0}\right)=1.01$. The results in Figs. 7 and 8 show that for even a small transverse initial momentum anisotropy, QGP-emitted photon $v_{2}$ at $p_{T} \gtrsim 2 \mathrm{GeV}$ can grow to more than 2 times higher values while the yield is essentially unchanged. We must note that in quantitative modeling of heavy-ion collisions, the proper way would be

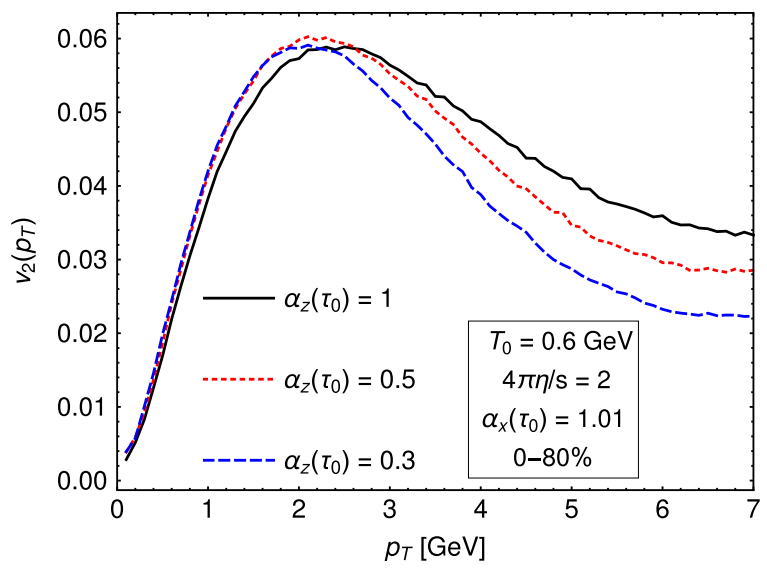

FIG. 7. Same as Fig. 6 but assuming a small initial transverse anisotropy $\alpha_{x}\left(\tau_{0}\right)=1.01$ of momentum distribution. 

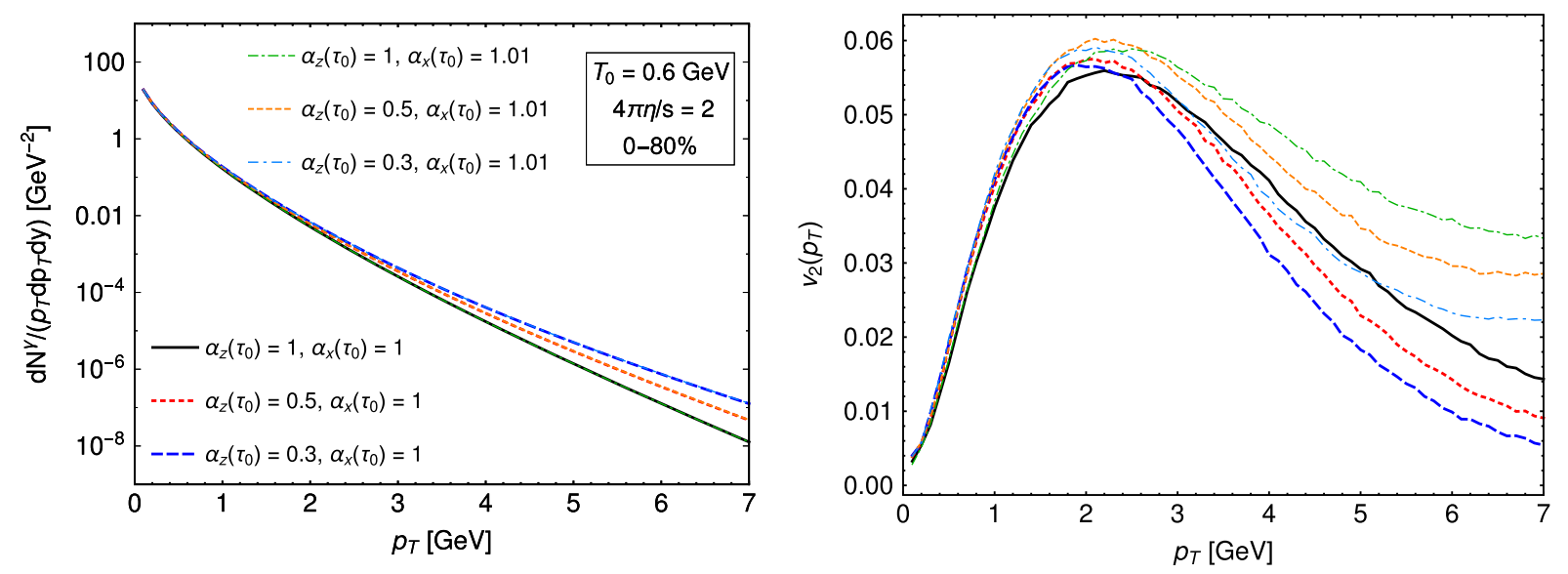

FIG. 8. The plots in Figs. 6 and 7 compared in the same figure.

to consider the strength and direction of initial transverse momentum anisotropy as fluctuating variables event by event. Therefore, the large sensitivity of the photon $v_{2}$ to $\alpha_{x}\left(\tau_{0}\right)$ as seen in the results of this paper must be considered as an overestimate and a motivation for future investigations including more relevant effects.

\section{Conformal approximation uncertainty}

In the calculations of photon yield and $v_{2}$ in this paper, when we convolve the photon rate with the aHydro results, we directly convert aHydro parameters $\left\{\alpha_{x, y, z}, \lambda\right\}$ to LRF photon rate parameters $\left\{\xi_{1,2}, \Lambda\right\}$ neglecting the temperature-dependent mass $\hat{m}$ which is present in aHydro evolution. In order to estimate the uncertainty due to this approximation, we rematch the aHydro effective temperature $T_{\text {eff }}\left(\lambda\left(\alpha_{x, y, z}, \hat{m}\right)\right)$ to the scale $\Lambda^{\prime}\left(\xi_{1,2}\right)$ defined by

$$
\begin{aligned}
\left(\frac{T_{\mathrm{eff}}}{\Lambda^{\prime}}\right)^{4}= & \frac{1}{4 \pi} \int_{0}^{2 \pi} d \phi \\
& \times \int_{-1}^{1} \frac{d(\cos \theta)}{\left(1+\xi_{1} \cos ^{2} \theta+\xi_{2} \sin ^{2} \theta \cos ^{2} \phi\right)^{2}},
\end{aligned}
$$

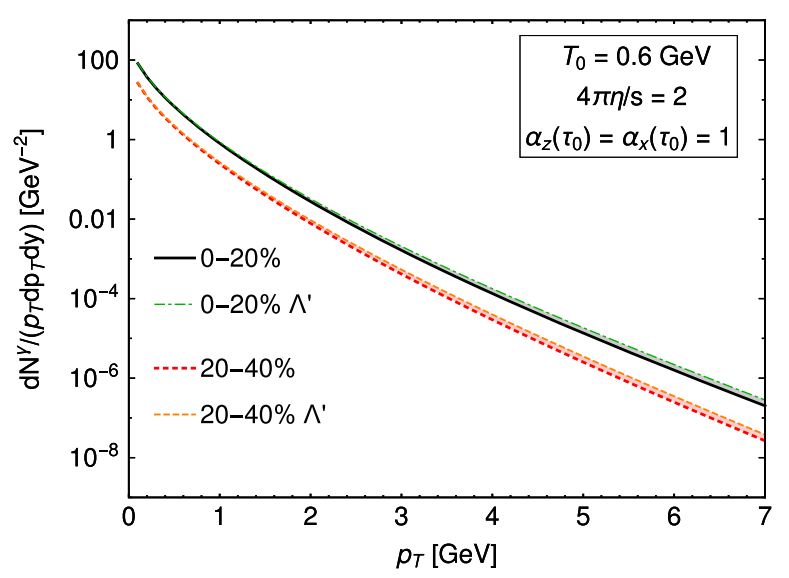

which is basically the matching of energy densities, i.e., $\epsilon\left(\Lambda^{\prime}\left(\xi_{1,2}\right)\right)=\epsilon_{\text {iso }}\left(T_{\text {eff }}\right)$. We repeat the calculations of photon yield and flow using $\Lambda^{\prime}\left(\xi_{1,2}\right)$ instead of $\lambda\left(\alpha_{x, y, z}, \hat{m}\right)$ and compare the results. The same calculations of the $0 \%-20 \%$ and $20 \%-40 \%$ centrality class presented in Fig. 4 are repeated with $\Lambda^{\prime}$ and compared in Fig. 9. The bands show the level of uncertainty due to conformal approximation. The effects of $\hat{m}$ on the yields and photon flow of central collisions seem to be negligible, and there is a notable uncertainty band for flow in the 20\%-40\% case. However, this uncertainty does not seem to change the overall estimates and qualitative interpretations of the results. In Fig. 10, we show the uncertainty band due to conformal approximation for the case with initial momentum anisotropy $\left\{\alpha_{z}\left(\tau_{0}\right)=0.5, \alpha_{x}\left(\tau_{0}\right)=1.01\right\}$ which seems to be small for both the photon yield and $v_{2}$ in the $0 \%-80 \%$ centrality class.

\section{E. Au-Au collisions at $\sqrt{\boldsymbol{s}}=\mathbf{2 0 0} \mathrm{GeV}$}

In all of the results presented in the previous sections, we used the aHydro model for $\mathrm{Pb}-\mathrm{Pb}$ collisions at the LHC at $\sqrt{s}=2.76 \mathrm{TeV}$. In this section, we show the results for the

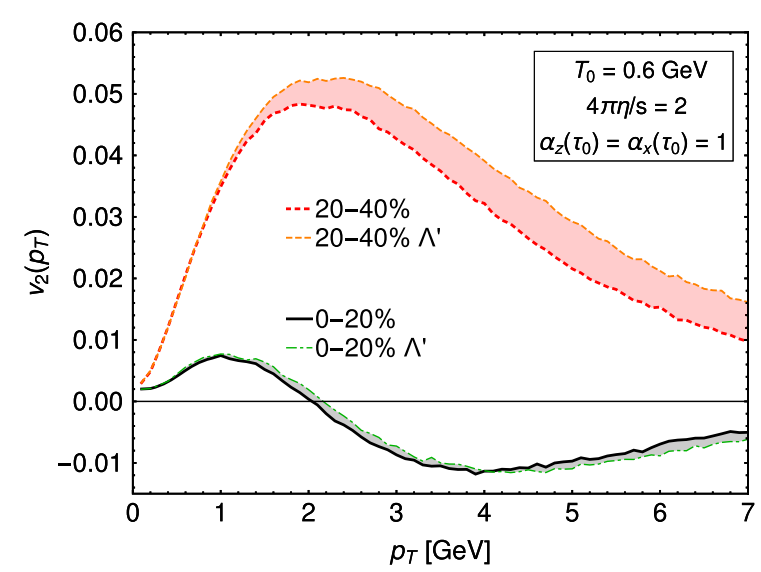

FIG. 9. Uncertainty bands due to conformal approximation neglecting $\hat{m}$ when connecting aHydro output with the LRF photon rate. Results are shown for initial momentum isotropic conditions $(\mathrm{Pb}-\mathrm{Pb})$. 

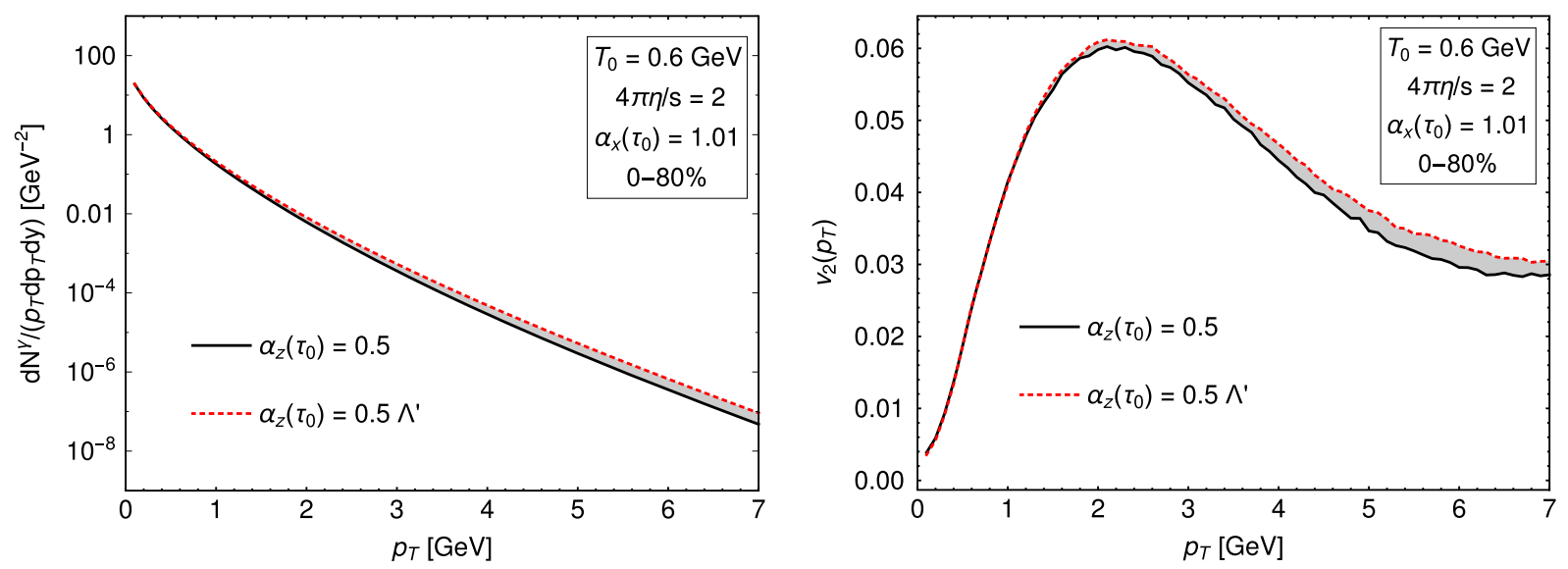

FIG. 10. Uncertainty bands due to conformal approximation for a case with initial momentum anisotropy ( $\mathrm{Pb}-\mathrm{Pb})$.
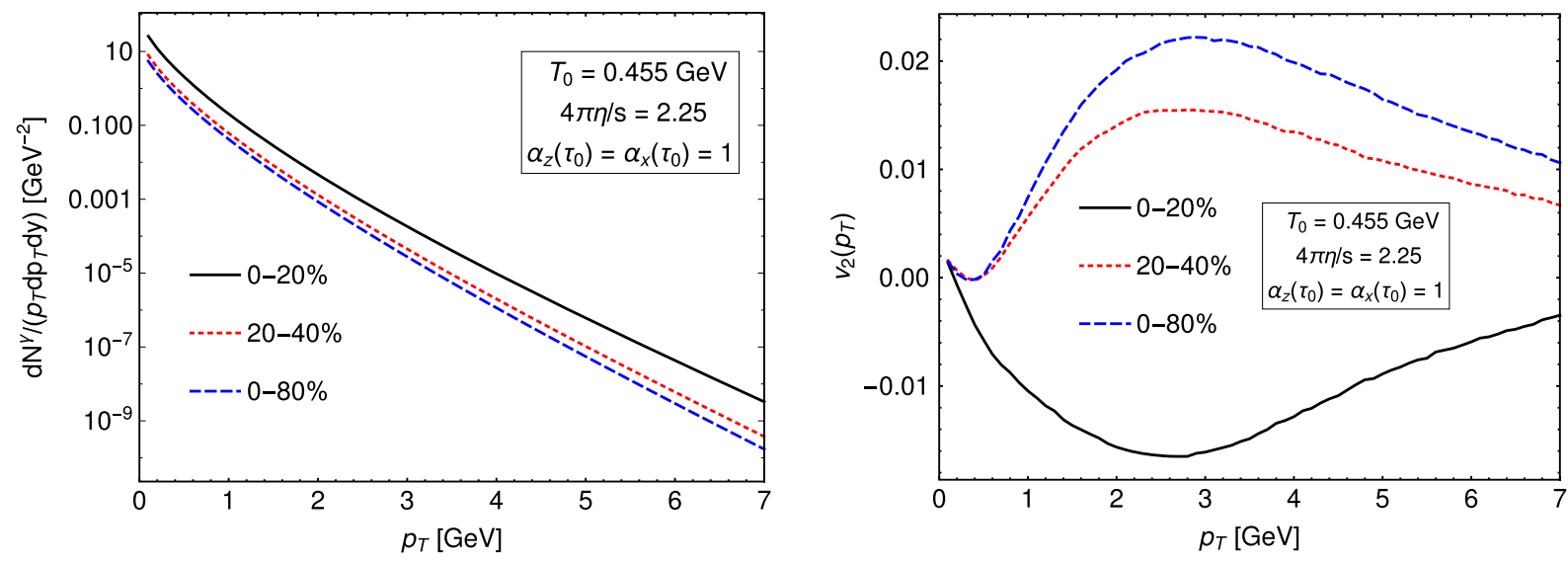

FIG. 11. Same calculations as Fig. 4 repeated for Au-Au collisions at the RHIC at $\sqrt{s}=200 \mathrm{GeV}$.

aHydro calculation of the yield and flow of photons emitted from QGP generated at $\mathrm{Au}-\mathrm{Au}$ collisions at the RHIC at $\sqrt{s}=200 \mathrm{GeV}$. We use the aHydro parameter values reported in [73] by tuning to the soft hadron spectra. The photon yield and $v_{2}$ results for three centrality classes

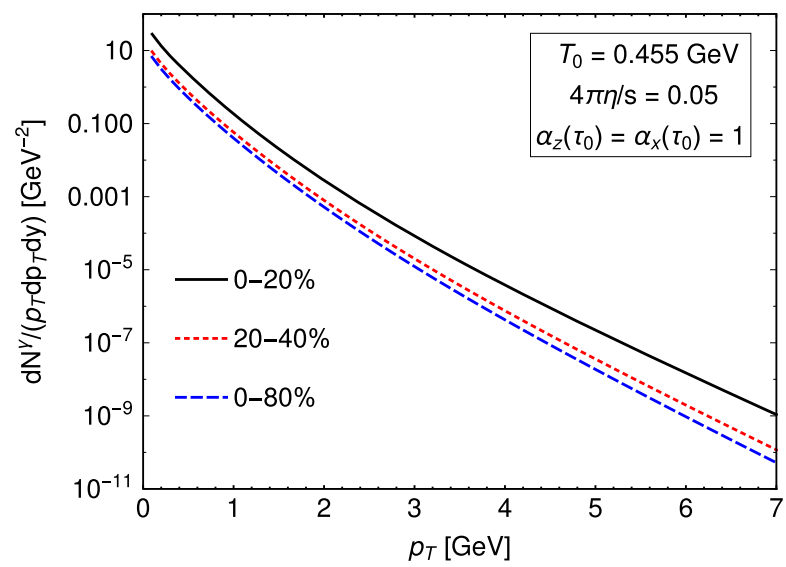

and momentum isotropic initial condition are shown in Fig. 11. Comparing with similar results for $\mathrm{Pb}-\mathrm{Pb}$ collisions in Fig. 4, it can be seen that, as expected, both yield and $v_{2}$ for the $\mathrm{Au}-\mathrm{Au}$ collision are smaller than in the $\mathrm{Pb}-\mathrm{Pb}$ case due to differences in $T_{0}, \eta / s$. In the aHydro simulations, the

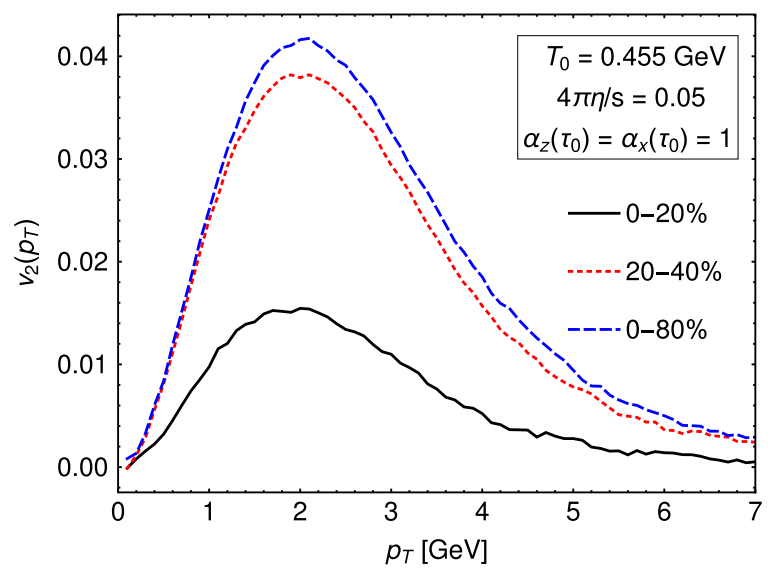

FIG. 12. Same calculations as Fig. 11 repeated for ideal (isotropic) limit of aHydro. 
lifetime of the system for $\sqrt{s}=200 \mathrm{GeV}$ is shorter than the system in $\sqrt{s}=2.76 \mathrm{TeV}$ collisions (by about $2.5 \mathrm{fm} / \mathrm{c}$ ). This means that the fluid generated at the $\sqrt{s}=$ $200 \mathrm{GeV}$ collisions does not have enough time to establish the same level of positive elliptic flow as in the $\mathrm{Pb}-\mathrm{Pb}$ collisions. In Fig. 12, we also repeat the simulations in the ideal (isotropic) limit of the hydrodynamic model for $\mathrm{Au}-\mathrm{Au}$ collisions at $\sqrt{s}=200 \mathrm{GeV}$. Similar to the case of $\mathrm{Pb}-\mathrm{Pb}$ collisions, the ideal limit of aHydro simulation for $\mathrm{Au}-\mathrm{Au}$ collisions results in a smaller yield but larger $v_{2}$ of photons compared to the full anisotropic simulation.

\section{CONCLUSIONS AND OUTLOOK}

In this paper, we calculated the real photon production in a momentum-anisotropic QGP. For the first time, we included transverse momentum anisotropy using an ellipsoidal parametrization of the QGP parton distribution functions. This is an extension of previous results of photon production including only longitudinal momentum anisotropy [51] and in line with our previous study of dilepton emission [87]. To calculate soft contributions to the photon rate, we utilized our previous results for quark self-energy in anisotropic QGP [81] advancing in the direction of including more information about collective excitations of the strongly interacting matter in phenomenological studies. In addition, the efficient method introduced in [81] for self-energy calculations in an anisotropic medium was essential in meeting the challenge of demanding numerical calculation of the photon rate with two extra parameters $\xi_{2}$ and $\phi_{q}$ compared to the previous spheroidally anisotropic case. We showed that the numerically calculated nonequilibrium photon rate can be accurately fitted to a function similar to the corresponding equilibrium rate by introducing direction dependence to its parameters. In particular, we introduced the anisotropic radiation temperature and radiation intensity for the QGP. We emphasize that these functions in general are not proportional to the anisotropic deformation introduced to the momentum distributions of QGP degrees of freedom. This idea provides us with a compact formulation of the photon rate which highly facilitates further calculations of yield and flow. We expect that similar ideas can be suitably applied to other observables such as dilepton production and modification of hard probes in nonequilibrium QGP.

We convolved the LRF anisotropic photon rate with space-time evolution of QGP modeled by aHydro using a quasiparticle EOS to obtain the yield and elliptic flow coefficient of zero rapidity real photons generated from the QGP in $\mathrm{Pb}-\mathrm{Pb}$ collisions at the LHC at $\sqrt{s}=2.76 \mathrm{TeV}$ and $\mathrm{Au}-\mathrm{Au}$ collision at the RHIC at $\sqrt{s}=200 \mathrm{GeV}$. With initially isotropic momentum distributions, we presented the results for the $0 \%-20 \%, 20 \%-40 \%$, and $0 \%-80 \%$ centrality classes. We observed that for central collisions the anisotropic model predicts negative $v_{2}$ for QGP generated photons. For $\mathrm{Pb}-\mathrm{Pb}$ collisions, we varied the initial momentum anisotropy and showed that for $p_{T} \gtrsim$ $2 \mathrm{GeV}$ the results are sensitive to initial longitudinal momentum anisotropy. We found a strong sensitivity of $v_{2}$ at $p_{T} \gtrsim 2 \mathrm{GeV}$ to the initial transverse momentum anisotropy. However, lacking a full analysis including event-by-event fluctuations and prompt photon sources, we expect that our results represent an overestimate of the effects of transverse initial momentum anisotropy. We also checked the uncertainty due to conformal approximation and neglecting the thermal quasiparticle mass in our calculations of the LRF rate. We showed that qualitative interpretations are not changed due to this uncertainty and overall estimates of the quantitative results can be considered reliable at this level.

In future studies, including a more complete set of photon sources in heavy-ion collisions such as prompt and hadronic gas and decay photons can provide a more definitive phenomenological understanding and better connection to experimental results. We expect that same ideas introduced in this paper can be useful for making further progress in incorporation of various contributions such as nonequilibrium, nonperturbative, and magnetic field effects in the photon production rate calculation.

\section{ACKNOWLEDGMENTS}

B.S.K. and M.S. were supported by the U.S. Department of Energy, Office of Science, Office of Nuclear Physics under Award No. DE-SC0013470.
[1] E. L. Feinberg, Nuovo Cimento Soc. Ital. Fis. 34A, 391 (1976).

[2] E. V. Shuryak, Yad. Fiz. 28, 796 (1978) [Phys. Lett. 78B, 150 (1978)].

[3] L. Oliva, M. Ruggieri, S. Plumari, F. Scardina, G. X. Peng, and V. Greco, Phys. Rev. C 96, 014914 (2017).
[4] I. Iatrakis, E. Kiritsis, C. Shen, and D.-L. Yang, J. High Energy Phys. 04 (2017) 035.

[5] G. David, Rep. Prog. Phys. 83, 046301 (2020).

[6] C. Gale, Proc. Sci., High-pT2017 (2019) 023 [arXiv:1802 .00128].

[7] A. Schäfer, J. M. Torres-Rincon, J. Rothermel, N. Ehlert, C. Gale, and H. Elfner, Phys. Rev. D 99, 114021 (2019). 
[8] S. Hauksson, S. Jeon, and C. Gale, Phys. Rev. C 97, 014901 (2018).

[9] C. Shen, J.-F. Paquet, U. Heinz, and C. Gale, Phys. Rev. C 91, 014908 (2015).

[10] S. Turbide, R. Rapp, and C. Gale, Phys. Rev. C 69, 014903 (2004).

[11] K. Dusling and I. Zahed, Phys. Rev. C 82, 054909 (2010).

[12] O. Linnyk, V. Konchakovski, T. Steinert, W. Cassing, and E. L. Bratkovskaya, Phys. Rev. C 92, 054914 (2015).

[13] G. Başar, D. Kharzeev, and V. Skokov, Phys. Rev. Lett. 109, 202303 (2012).

[14] B. Müller, S.-Y. Wu, and D.-L. Yang, Phys. Rev. D 89, 026013 (2014).

[15] H. van Hees, C. Gale, and R. Rapp, Phys. Rev. C 84, 054906 (2011).

[16] J. I. Kapusta, P. Lichard, and D. Seibert, Phys. Rev. D 44, 2774 (1991); 47, 4171(E) (1993).

[17] H. Nadeau, J. I. Kapusta, and P. Lichard, Phys. Rev. C 45, 3034 (1992).

[18] M. Heffernan, P. Hohler, and R. Rapp, Phys. Rev. C 91, 027902 (2015).

[19] V. Khachatryan, B. Schenke, M. Chiu, A. Drees, T. K. Hemmick, and N. Novitzky, Nucl. Phys. A978, 123 (2018).

[20] A. Monnai, J. Phys. G 47, 075105 (2020).

[21] A. Monnai, Phys. Rev. C 92, 014905 (2015).

[22] A. Monnai, Phys. Rev. C 90, 021901 (2014).

[23] S. Benić, K. Fukushima, O. Garcia-Montero, and R. Venugopalan, J. High Energy Phys. 01 (2017) 115.

[24] K. Fukushima and K. Mameda, Phys. Rev. D 86, 071501 (2012).

[25] J. Jalilian-Marian, Nucl. Phys. A753, 307 (2005).

[26] J. Berges, K. Reygers, N. Tanji, and R. Venugopalan, Phys. Rev. C 95, 054904 (2017).

[27] R. J. Fries, B. Müller, and D. K. Srivastava, Phys. Rev. C 72, 041902 (2005).

[28] F. D. Steffen and M. H. Thoma, Phys. Lett. B 510, 98 (2001); 660, 604(E) (2008).

[29] H. van Hees, M. He, and R. Rapp, Nucl. Phys. A933, 256 (2015).

[30] L. McLerran and B. Schenke, Nucl. Phys. A946, 158 (2016).

[31] A. Ayala, J. D. Castaño-Yepes, C. A. Dominguez, L. A. Hernández, S. Hernández-Ortiz, and M. E. TejedaYeomans, Phys. Rev. D 96, 014023 (2017); 96, 119901(E) (2017).

[32] R. J. Fries, B. Müller, and D. K. Srivastava, Phys. Rev. Lett. 90, 132301 (2003).

[33] J. Ghiglieri, J. Hong, A. Kurkela, E. Lu, G. D. Moore, and D. Teaney, J. High Energy Phys. 05 (2013) 010.

[34] J. J. Neumann, D. Seibert, and G. I. Fai, Phys. Rev. C 51, 1460 (1995).

[35] D. K. Srivastava, Eur. Phys. J. C 10, 487 (1999); 20, 399(E) (2001).

[36] A. Dumitru, U. Katscher, J. A. Maruhn, H. Söcker, W. Greiner, and D. H. Rischke, Phys. Rev. C 51, 2166 (1995).

[37] R. Baier, M. Dirks, K. Redlich, and D. Schiff, Phys. Rev. D 56, 2548 (1997).

[38] P. B. Arnold, G. D. Moore, and L. G. Yaffe, J. High Energy Phys. 12 (2001) 009.

[39] C. M. Hung and E. V. Shuryak, Phys. Rev. C 56, 453 (1997).
[40] S. S. Singh and Y. Kumar, Int. J. Mod. Phys. A 30, 1550020 (2015).

[41] K. Tuchin, Phys. Rev. C 91, 014902 (2015).

[42] J. Ghiglieri, O. Kaczmarek, M. Laine, and F. Meyer, Phys. Rev. D 94, 016005 (2016).

[43] L. D. McLerran and T. Toimela, Phys. Rev. D 31, 545 (1985).

[44] M. Strickland, Phys. Lett. B 331, 245 (1994).

[45] T. Sakaguchi, Pramana 84, 845 (2015).

[46] Y. Hidaka, S. Lin, R. D. Pisarski, and D. Satow, J. High Energy Phys. 10 (2015) 005.

[47] G. Başar, D. E. Kharzeev, and E. V. Shuryak, Phys. Rev. C 90, 014905 (2014).

[48] S. Turbide, C. Gale, and R. J. Fries, Phys. Rev. Lett. 96, 032303 (2006).

[49] G. Baym, T. Hatsuda, and M. Strickland, Phys. Rev. C 95, 044907 (2017).

[50] L. Ruan, Nucl. Phys. A931, 185 (2014).

[51] B. Schenke and M. Strickland, Phys. Rev. D 76, 025023 (2007).

[52] R. Chatterjee, E. S. Frodermann, U. W. Heinz, and D. K. Srivastava, Phys. Rev. Lett. 96, 202302 (2006).

[53] R. Chatterjee, H. Holopainen, I. Helenius, T. Renk, and K. J. Eskola, Phys. Rev. C 88, 034901 (2013).

[54] P. Dasgupta, R. Chatterjee, S. K. Singh, and J.-E. Alam, Phys. Rev. C 97, 034902 (2018).

[55] L. Bhattacharya, R. Ryblewski, and M. Strickland, Phys. Rev. D 93, 065005 (2016).

[56] J.-F. Paquet, C. Shen, G. S. Denicol, M. Luzum, B. Schenke, S. Jeon, and C. Gale, Phys. Rev. C 93, 044906 (2016).

[57] C. Shen, U. W. Heinz, J.-F. Paquet, I. Kozlov, and C. Gale, Phys. Rev. C 91, 024908 (2015).

[58] M. Dion, J.-F. Paquet, B. Schenke, C. Young, S. Jeon, and C. Gale, Phys. Rev. C 84, 064901 (2011).

[59] Y.-M. Kim, C.-H. Lee, D. Teaney, and I. Zahed, Phys. Rev. C 96, 015201 (2017).

[60] H. Holopainen, S. Räsänen, and K. J. Eskola, Phys. Rev. C 84, 064903 (2011).

[61] G. Vujanovic, J.-F. Paquet, G. S. Denicol, M. Luzum, S. Jeon, and C. Gale, Phys. Rev. C 94, 014904 (2016).

[62] C. Gale, Y. Hidaka, S. Jeon, S. Lin, J.-F. Paquet, R. D. Pisarski, D. Satow, V. V. Skokov, and G. Vujanovic, Phys. Rev. Lett. 114, 072301 (2015).

[63] A. Adare et al. (PHENIX Collaboration), Phys. Rev. Lett. 123, 022301 (2019).

[64] V. Khachatryan (PHENIX Collaboration), Nucl. Phys. A982, 763 (2019).

[65] A. Adare et al. (PHENIX Collaboration), Phys. Rev. C 94, 064901 (2016).

[66] S. Acharya et al. (ALICE Collaboration), Phys. Lett. B 789, 308 (2019).

[67] M. Strickland, Acta Phys. Pol. B 45, 2355 (2014).

[68] W. Florkowski and R. Ryblewski, Phys. Rev. C 83, 034907 (2011).

[69] M. Martinez and M. Strickland, Nucl. Phys. A848, 183 (2010).

[70] M. Alqahtani, M. Nopoush, R. Ryblewski, and M. Strickland, Phys. Rev. C 96, 044910 (2017).

[71] M. Alqahtani, M. Nopoush, R. Ryblewski, and M. Strickland, Phys. Rev. Lett. 119, 042301 (2017). 
[72] M. Alqahtani, M. Nopoush, and M. Strickland, Prog. Part. Nucl. Phys. 101, 204 (2018).

[73] D. Almaalol, M. Alqahtani, and M. Strickland, Phys. Rev. C 99, 044902 (2019).

[74] W. Florkowski, E. Maksymiuk, R. Ryblewski, and M. Strickland, Phys. Rev. C 89, 054908 (2014).

[75] M. Nopoush, R. Ryblewski, and M. Strickland, Phys. Rev. C 90, 014908 (2014).

[76] L. Tinti, R. Ryblewski, W. Florkowski, and M. Strickland, Nucl. Phys. A946, 29 (2016).

[77] M. Strickland, M. Nopoush, and R. Ryblewski, Nucl. Phys. A956, 268 (2016).

[78] M. Strickland, J. Noronha, and G. Denicol, Phys. Rev. D 97 , 036020 (2018).

[79] M. Strickland, J. High Energy Phys. 12 (2018) 128.
[80] P. Romatschke and M. Strickland, Phys. Rev. D 68, 036004 (2003).

[81] B. S. Kasmaei and M. Strickland, Phys. Rev. D 97, 054022 (2018).

[82] E. Braaten and R. D. Pisarski, Nucl. Phys. B337, 569 (1990).

[83] S. Mrówczyński and M.H. Thoma, Phys. Rev. D 62, 036011 (2000).

[84] B. S. Kasmaei, M. Nopoush, and M. Strickland, Phys. Rev. D 94, 125001 (2016).

[85] B. Schenke and M. Strickland, Phys. Rev. D 74, 065004 (2006).

[86] E. Braaten and T. C. Yuan, Phys. Rev. Lett. 66, 2183 (1991).

[87] B. S. Kasmaei and M. Strickland, Phys. Rev. D 99, 034015 (2019). 